\title{
Epidémiologie de la dysfonction érectile (2ème partie). Facteurs de risque
}

\author{
Dominique DELAVIERRE \\ Service d'Uro-Andrologie, CHR La Source, Orléans
}

RESUME

Les enquêtes épidémiologiques réalisées dans la population générale de nombreux pays et l'étude de populations ciblées (dont un exemple est l'enquête réalisée en France par Leriche et Jaudinot chez 7469 patients diabétiques, hypertendus ou les deux) ont mis en évidence de nombreux facteurs de risque de dysfonction érectile : diabète, hypertension artérielle, tabagisme, dyslipidémie, maladies cardio-vasculaires, troubles psychologiques, certains médicaments, insuffisance rénale chronique, facteurs liés au statut socio-économique et au mode de vie, obésité, symptômes du bas appareil urinaire, mauvais état général et cyclisme.

Les facteurs de risque cardio-vasculaires sont prédictifs de dysfonction érectile et actuellement la dysfonction érectile est souvent considérée comme une entité de la maladie vasculaire ou endothéliale.

De nouvelles études sont nécessaires pour préciser les mécanismes physiopathologiques de certains facteurs de risque et l'intérêt éventuel de mesures de prévention.

Mots clés : épidémiologie, prévalence, dysfonction érectile, impuissance sexuelle
Le 1er chapitre de notre travail (voir Andrologie 12, $2: 167-185$ ) concernait la fréquence (prévalence et incidence) de la dysfonction érectile dans des populations définies (épidémiologie descriptive). Cette $2^{\text {ème }}$ partie s'intéresse aux relations entre la dysfonction érectile et différents facteurs de risque (épidémiologie analytique). La dysfonction érectile est d'ailleurs (comme les maladies cardio-vasculaires) un terrain de prédilection pour l'épidémiologie analytique.

En épidémiologie, un risque est la probabilité de survenue d'un événement, et un facteur de risque une caractéristique en fonction de laquelle varie ce risque. L'odds-ratio (OR) apprécie l'importance relative de deux risques. L'odds-ratio ajusté (OR ajusté) est l'estimation d'un oddsratio lié à un facteur donné en tenant compte d'autres facteurs.

Dans le domaine de la dysfonction érectile, la notion de facteurs de risque s'est imposée ces dernières années pour de multiples raisons : i) constatation du caractère souvent multifactoriel de la dysfonction érectile et de l'intrication des facteurs étiologiques ; ii) meilleure approche physiopathologique de la dysfonction érectile (souvent assimilée à une maladie vasculaire) ; iii) publication d'enquêtes épidémiologiques récentes très documentées.

\section{Correspondance :}

Dr Dominique DELAVIERRE - Service d'Uro-Andrologie. CHR La Source, BP 6709, 45067 ORLEANS Cedex 2 - Tel 0238 514626 - Fax 0238514159 - Email delav.uro@wanadoo.fr 


\section{MATERIEL ET METHODES}

Deux méthodes permettent de recueillir des données :

1) les enquêtes épidémiologiques réalisées dans la population générale (décrites dans la 1ère partie) notamment les enquêtes américaines MMAS et NHSLS, australiennes de Chew et Pinnock, italienne de Parazzini, espagnole de Martin-Morales et allemande de Braun. Ces enquêtes permettent de comparer la prévalence de la dysfonction érectile dans les populations à risque et générale, et précisent les odds-ratios (OR) [7, 11, 17, $30,38,42,43]$;

2) l'étude de populations ciblées dont un exemple est l'enquête réalisée en France par Leriche et Jaudinot : d'octobre 1999 à février 2000, 1845 médecins généralistes ont proposé à 7469 patients d'âge moyen 58,9 ans, diabétiques $(31,8 \%)$, hypertendus $(52,3 \%)$ ou les deux $(15,9 \%)$, des questionnaires sur leur fonction érectile (perception par le patient de sa fonction érectile et IIEF 5) [32].

Ces différentes enquêtes ont permis la mise en évidence de nombreux facteurs de risque : diabète, hypertension artérielle, tabagisme, dyslipidémie, maladies cardio-vasculaires, troubles psychologiques, certains médicaments, insuffisance rénale chronique, facteurs liés au statut socio-économique et au mode de vie, obésité, symptômes du bas appareil urinaire, mauvais état général et cyclisme.

\section{FACTEURS DE RISQUE}

\section{Diabète}

a) Prévalence de la dysfonction érectile

Les différentes enquêtes publiées récemment permettent de chiffrer la prévalence de la dysfonction érectile chez les diabétiques entre 20 et $67,4 \%$ (Tableau 1 ).

Dans les enquêtes réalisées dans la population générale la prévalence de la dysfonction érectile est toujours significativement plus élevée chez les diabétiques que dans cette population générale : dans l'enquête MMAS initiale, $28 \%$ après ajustement sur l'âge contre $9,6 \%$ (en ce qui concerne la dysfonction érectile sévère), dans celles de Chew et Parazzini respectivement 34,4 et $48,6 \%$ contre 18,6 et $12,8 \%[11,17,42]$.

Dans les enquêtes ciblées cette prévalence est variable mais toujours élevée voire très élevée. Klein a mené une enquête dans le Wisconsin chez 359 patients (âgés d'au moins 21 ans mais de moins de 30 ans lors de la découverte du diabète, diabétiques insulino-dépendants depuis au moins 10 ans) et chiffre la prévalence de la dysfonction érectile à 20\% [29]. Cette prévalence est beaucoup plus élevée dans l'enquête de Siu conduite à Hong Kong (63,6 $\%)$ chez 486 diabétiques [47] ou dans celle de Leriche et Jaudinot $(67,4 \%)$ [32].

Selon Sairam, il est possible de découvrir un diabète au décours d'une dysfonction érectile. Dans sa série de 129 patients insuffisants érectiles 27 étaient diabétiques dont 5 l'ignoraient [45].

\section{b) Incidence}

L'enquête de Johannes (MMAS), détaillée dans la 1ère partie de notre article (voir Andrologie 12, 2 :167-185), chiffre l'incidence de la dysfonction érectile chez les diabétiques à 50,7 pour 1000 par an contre 25,9 dans la population générale avec un risque relatif après ajustement sur l'âge de 1,83 [27]. En Italie, Fedele a suivi 1010 diabétiques pendant 2,8 ans en moyenne et chiffre l'incidence de la dysfonction érectile à 68 pour 1000 par an (intervalle de confiance à 95\%: 59-77) [15].

\section{c) Influence de l'âge}

La prévalence de la dysfonction érectile passe, dans l'enquête de Klein, de $1,1 \%$ dans la tranche 21 à 30 ans à 47,1 $\%$ après 42 ans [29], et dans celle de Siu de 33,3\% à 21 ans à 73,8 \% à 80 ans [47]. Dans l'enquête de Fedele, l'in-

Tableau 1 : Prévalence de la dysfonction érectile chez les diabétiques.

\begin{tabular}{llll}
\hline & Auteur (année) & Prévalence & OR ou OR ajusté \\
\hline \multirow{3}{*}{ Enquêtes réalisées dans } & Feldman (MMAS) (1994) [17] & $28 \%$ & - \\
la population générale & Parazzini (2000) [42] & $48,6 \%$ & 3,6 \\
& Chew (2000) [11] & $34,4 \%$ & 2,53 \\
& Braun (2000) [7] & - & 3,95 \\
& Martin-Morales (2001) [38] & - & 4,08 (Question générale) \\
Enquêtes ciblées & Klein (1996) [29] & & 1,98 (IIEF) \\
& Fedele (1998) [16] & $20 \%$ & - \\
& Leriche/Jaudinot (2000) [32] & $36 \%$ & - \\
& Siu (2001) [47] & $67,4 \%$ & - \\
\hline
\end{tabular}


cidence passe de 15 pour 1000 par an dans la tranche19 à 29 ans à 149 dans la tranche 70 à 79 ans [15]. Selon Siu, la sévérité de la dysfonction érectile est également influencée par l'âge : nulle avant 40 ans, la prévalence de la dysfonction érectile sévère passe de $7,4 \%$ à 41 ans à $71,1 \%$ à 80 ans [47].

\section{d) Facteurs de risque}

La prévalence et l'incidence de la dysfonction érectile chez les diabétiques sont plus élevées en présence d'autres facteurs de risque. Ainsi, cette prévalence passe dans l'enquête de Leriche et Jaudinot à $77,9 \%$ chez les diabétiques hypertendus [32]. L'influence de l'hypertension artérielle est également soulignée par Parazzini $(\mathrm{OR}=8,1)$ [42] et Fedele [15].

Bortolotti, au cours d'une enquête menée en Italie chez 9670 diabétiques âgés de 20 à 70 ans, chiffre les OR ajustés à 1,4 chez les fumeurs et 1,5 chez les anciens fumeurs en référence aux non-fumeurs et relève, en tenant compte de l'âge, une aggravation du risque de dysfonction érectile avec l'intensité et l'ancienneté d'un éventuel tabagisme associé. Chez les anciens fumeurs ce risque diminue significativement avec l'éloignement de l'arrêt du tabagisme en nombre d'années [6].

Selon Klein, après ajustement sur l'âge, 3 facteurs de risque augmentent la prévalence de la dysfonction érectile: une $\mathrm{HbA} 1 \mathrm{c} \geq 10,9 \%$ comparée à un chiffre $<8,8 \%(\mathrm{OR}=$ $6,25)$, un indice de masse corporelle $\geq 31,1$ comparé à un chiffre $<26(\mathrm{OR}=2,1)$, et la prise de diurétiques [29].

Fedele confirme l'incidence plus élevée de la dysfonction érectile avec une $\mathrm{HbA} 1 \mathrm{c} \geq 9 \%$ (comparée à un chiffre < $7,5 \%)$ et une ancienneté du diabète supérieure à 10 ans (comparée à moins de 5 ans) [15]. Siu relève également l'influence de l'ancienneté du diabète : la prévalence de la dysfonction érectile passe de $56 \%$ quand le diabète évolue depuis moins de 5 ans à $72 \%$ quand il évolue depuis plus de 20 ans [47]. Toutefois, après ajustement sur l'âge, Klein estime que l'ancienneté du diabète n'est pas un facteur de risque, pas plus d'ailleurs que l'hypertension artérielle ou le tabagisme [29].

\section{e) Complications associées}

Les enquêtes de Fedele, Klein ou Siu montrent que la dysfonction érectile est significativement associée chez les diabétiques à d'autres complications : neuropathie, angiopathie des membres inférieurs, rétinopathie, néphropathie, cardiopathie ischémique $[15,29,47]$.

\section{f) Physiopathologie}

La dysfonction érectile est liée non seulement aux classiques angiopathie et neuropathie mais aussi à une altération directe de la relaxation des fibres musculaires lisses des corps caverneux neuromédiée et endothélo-dépendante [25]. L'impact psychologique de la maladie n'est pas négligeable non plus.

\section{Hypertension artérielle}

\section{a) Prévalence de la dysfonction érectile}

Les différentes enquêtes publiées récemment permettent de chiffrer la prévalence de la dysfonction érectile chez les hypertendus entre 15 et $68,3 \%$ (Tableau 2).

Dans les enquêtes réalisées dans la population générale, la prévalence de la dysfonction érectile est toujours significativement plus élevée chez les hypertendus que dans cette population générale (sauf dans l'enquête de Chew) : dans l'enquête MMAS initiale, $15 \%$ après ajustement sur l'âge contre $9,6 \%$ (en ce qui concerne la dysfonction érectile sévère), et dans celle de Parazzini, 30,1\% contre $12,8 \%$ $(\mathrm{OR}=1,6)[17,42]$.

Dans les enquêtes ciblées de Jensen (101 patients) [26], Burchardt (104 patients) [8], et Leriche et Jaudinot (3906 patients) [32], cette prévalence est respectivement de 27 , 68,3 et $60,9 \%$.

\section{b) Incidence}

L'enquête de Johannes (MMAS) chiffre l'incidence de la dysfonction érectile chez les hypertendus à 42,5 pour 1000 par an contre 25,9 dans la population générale avec un risque relatif après ajustement sur l'âge de 1,52 [27].

Tableau 2 : Prévalence de la dysfonction érectile chez lez hypertendus.

\begin{tabular}{lllc}
\hline & Auteur (année) & Prévalence & OR ou OR ajusté \\
\hline & Feldman (MMAS) (1994) [17] & $15 \%$ & - \\
Enquêtes réalisées dans & Parazzini (2000) [42] & $30,1 \%$ & 1,6 \\
la population générale & Braun (2000) [7] & - & 1,58 \\
& Martin-Morales (2001) [38] & - & 1,58 (Question générale) \\
& & & - \\
Enquêtes ciblées & Jensen (1999) [26] (IIEF) & $27 \%$ & - \\
& Leriche/Jaudinot (2000) [32] & $60,9 \%$ & -
\end{tabular}




\section{c) Complications associées}

Dans la série de Jensen, la dysfonction érectile est significativement associée à la claudication intermittente et à la cardiopathie ischémique [26].

\section{d) Physiopathologie}

Les travaux récents de Behr-Roussel et Giuliano, chez des rats spontanément hypertendus, démontrent expérimentalement l'influence de l'hypertension artérielle sur la fonction érectile. La réponse érectile (évaluée par la mesure de la pression intra-caverneuse lors de stimulations électriques du nerf caverneux) est significativement diminuée chez les rats hypertendus comparativement à des rats témoins normotendus [5].

Jensen évoque l'altération certainement athéroscléreuse des artères de la verge et le rôle de la baisse de la pression artérielle lors de la mise en route d'un traitement hypertenseur : dans sa série, 12 dysfonctions érectiles sur 27 sont apparues lors de la mise en route d'un tel traitement [26]. Nous verrons toutefois au chapitre des médicaments que ces conclusions ne sont pas confirmées par d'autres études.

\section{Tabagisme}

\section{a) Prévalence de la dysfonction érectile}

Tengs a réalisé une méta-analyse de 19 études publiées de 1980 à 2001, regroupant 3819 patients insuffisants érectiles. Dans 16 études, la prévalence du tabagisme est supérieure à celle de la population générale. Ainsi selon cette méta-analyse $40 \%$ des insuffisants érectiles sont fumeurs contre $28 \%$ dans la population générale [50]. Mannino publie en 1994 une enquête réalisée chez 4462 vétérans du Vietnam âgés de 31 à 49 ans. La prévalence de la dysfonction érectile est de $3,7 \%$ chez les fumeurs contre $2,2 \%$ chez les non-fumeurs et $2 \%$ chez les anciens fumeurs (OR ajusté $=1,5)[36]$.

Le tabagisme est également considéré comme un facteur de risque dans les enquêtes de Martin-Morales (au delà de 40 cigarettes par jour, OR ajusté $=2,5$ avec la question générale et 1,59 avec l'IIEF) [38] et dans celle de Parazzini ( $8 \%$ de dysfonction érectile che $z$ les non-fumeurs contre $13,3 \%$ chez les fumeurs - OR $=1,7$ - et $19,4 \%$ chez les anciens fumeurs - $\mathrm{OR}=1,6)[42]$, mais pas dans celle de Braun [7].

Dans l'enquête MMAS initiale, le tabagisme n'était pas non plus considéré comme un facteur de risque indépendant mais potentialisait d'autres facteurs de risque : maladies cardiovasculaires traitées, hypertension artérielle traitée, traitements cardiaques, antihypertenseurs et vasodilatateurs [17]. Toutefois la 2ème enquête réalisée 8 ans plus tard apporte des conclusions différentes. Feldman étudie 513 hommes initialement sans dysfonction érectile ni diabète ni maladies cardiaques et sans traitement. Il évalue dans la 2 ème enquête la prévalence de la dysfonction érectile dans cette population en fonction du statut tabagique initial. Cette prévalence est de $24 \%$ chez les fumeurs contre $14 \%$ chez les non-fumeurs et $14 \%$ chez les anciens fumeurs (OR =1,97). Le tabagisme passif au travail ou à la maison est également un facteur de risque significatif (OR $=2,45)$ de même que fumer des cigares $(O R=2,07)$ [ 18$]$.

\section{b) Facteurs de risque}

Selon Parazzini, 1'ancienneté et l'importance du tabagisme sont des facteurs de risque : au delà de 20 ans de tabagisme, la prévalence de la dysfonction érectile est de $23,5 \%$ contre $7,6 \%$ en deçà $(O R=1,6)$; à partir de 10 cigarettes par jour, elle est de $23,5 \%$ contre $16,2 \%$ en dessous (OR $=1,6)[42]$. Toutefois, Mannino ne confirme pas l'influence de l'ancienneté et de l'importance du tabagisme [36].

\section{c) Influence de l'arrêt du tabagisme}

Selon Guay l'arrêt du tabagisme améliore rapidement et significativement la qualité des érections dans le 1er mois [24]. Les enquêtes de Mannino [36] et Feldman [18] montrent également que la prévalence du tabagisme est identique chez les anciens fumeurs et les non-fumeurs, mais pas celle de Parazzini [42].

\section{d) Physiopathologie}

Des études expérimentales humaines et animales démontrent que le tabagisme altère la relaxation des fibres musculaires lisses des corps caverneux neuromédiée et endothélo-dépendante mais le mécanisme de cette altération est incumbu i34j.

\section{Dyslipidémie}

L'enquête MMAS initiale montre que la baisse du taux de cholestérol HDL de 0,9 à $0,3 \mathrm{~g} / 1$ augmente la prévalence de la dysfonction érectile modérée de 6,7 à $25 \%$ dans la tranche 40 à 55 ans et sévère de 0 à $16 \%$ dans la tranche 56 à 70 ans [17]. Selon Martin-Moralès, l'hypercholestérolémie totale est un facteur de risque significatif (OR ajusté $=1,63$ avec la question générale et 1,62 avec l'IIEF) [38] ; il en est de même pour Pinnock, après ajustement sur l'âge, pour l'hypertriglycéridémie [43], mais pas pour Parazzini [42].

A Dallas, Wei a mené une étude prospective chez 3250 hommes, âgés en moyenne de 51 ans, sans dysfonction érectile. Au cours d'un suivi moyen de 22 mois, 71 hommes ont développé une dysfonction érectile. Après ajustement sur l'âge, l'hypercholestérolémie totale et la baisse du taux de cholestérol HDL apparaissent dans cette étude comme des facteurs de risque de dysfonction érectile [51].

Ces différentes enquêtes indiquent que l'hypercholestćrolémie totale, la baisse du cholestérol HDL et l'hypertriglycéridémie augmentent le risque de dysfonction érectile 
mais par contre il n'est pas prouvé que corriger la dyslipidémie réduise le risque de développer une dysfonction érectile, d'autant que les statines et surtout les fibrates sont soupçonnés d'être délétères pour l'érection [46]. Des études sont nécessaires pour établir les bénéfices des statines et des fibrates à court ou long terme dans le traitement et la prévention de la dysfonction érectile [46].

\section{Maladies cardio-vasculaires}

Les facteurs de risque cardio-vasculaires sont prédictifs de dysfonction érectile, et la prévalence de la dysfonction érectile est plus élevée lors de cardiopathie notamment ischémique et de pathologie vasculaire périphérique [2], $38,48,49]$. Ainsi la prévalence de la dysfonction érectile sévère est, dans l'enquête MMAS initiale, de $39 \%$ chez les malades cardio-vasculaires traités, après ajustement sur l'âge, contre 9,6\% dauns la population générale [17]; dans celle de Chew, la prévalence est de $38,1 \%(\mathrm{OR}=1,87)$ lors de cardiopathie ischémique et de $56,8 \%$ (OR $=5,13$ ) lors de pathologie vasculaire périphérique contre $18,6 \%$ dans la population génćrale [11]. Dans l'enquête de Parazzini, la prévalence de la dysfonction érectile est de $33,7 \%$ lors de pathologie cardiovasculaire contre $12,8 \%$ dans la population générale $(\mathrm{OR}=2,2)$ |42].

Au niveau des corps caverneux, la relaxation des fibres musculaires lisses et le remplissage des espaces sinusoïdes (théorie de l'éponge active de Bondil) nécessitent un apport artériel suffisant et un fonctionnement satisfaisant de l'endothélium qui recouvre ces espaces (libération de monoxyde d'azote N(). La dysfonction érectile est donc très souvent une pathologie artérielle ou endothéliale et s'inscrit dans le cadre de la maladie vasculaire ou maladie endothéliale.

Toutefois la dysfonction érectile n'est pas seulement une entité de la maladie cndothéliale, elle est aussi prédictive d'autres localisations de cette maladie et notamment de coronaropathie [28, 41]. La dysfonction érectile est donc un marqueur cardio-vasculaire et un signal d'alerte.

\section{Troubles psychologiques}

De nombreuses situations psychopathologiques favorisent la survenue d'une dystonction érectile : anxiété, angoisse de performance, dépression (dans l'enquête MMAS initiale, la prévalence de la dysfonction érectile modérée ou sévère est de presque $90 \%$ lors de dépression sévère, 59 $\%$ lors de dépression modérée et $25 \%$ lors de dépression mineure) [17], stress, colère exprimée ou réprimée, personnalité soumise (alors qu'une personnalité dominante protège, comme l'atteste l'enquêtc MMAS initiale) [2, 17], problèmes existentiels et environnementaux (conjugaux, familiaux, financiers, professionnels), défaut d'information ou ignorance de la sexualité, et antécédents d'abus sexuels par des adultes dans l'enfance [30].
Lee insiste sur l'intérêt d'un bilan psychologique spécialisé. Dans sa série de 120 patients, il en dénombre 40 présentant des facteurs de risque psychopathologiques (dépression, dépendance à l'alcool ou à une drogue, schizophrénie ou personnalité schizoïde, anxiété, TOC), alors qu'un ter bilan urologique en avait dépisté uniquement 16 [31].

La dysfonction érectile elle-même entraîne des perturbations psychologiques, ce qui explique la fréquente intrication des facteurs étiologiques par installation d'un cercle vicieux.

\section{Médicaments}

La responsabilité des médicaments dans la survenue d'une dysfonction érectile est souvent difficile à affirmer d'autant que certains d'entre eux traitent des pathologies favorisant la dysfonction érectile (hypertension artérielle, dyslipidémie ou dépression notamment).

De nombreux médicaments sont incriminés dans diverses enquêtes $[4,13,17,38,39)$ : psychotropes, somnifères, benzodiazépines, hypoglycémiants, antihypertenseurs (surtout bêtabloquants, diurétiques thiazidiques ou non, et spironolactone car les inhibiteurs calciques ct les inhibiteurs de l'enzyme de conversion sont moins impliqués, les alphabloquants semblant même avoir un effet plutôt bénéfique), digitaliques, dérivés nitrés, vasodilatateurs, antihistaminiques $\mathrm{H} 2$, hormones, anticholinergiques, agents cytotoxiques, statines et fibrates.

Toutefois, l'interprétation de ces enquêtes est difficile compte-tenu de l'existence de co-morbidités et de problèmes de santé généraux. Derby a étudié une cohorte de 1476 hommes (enquête initiale) puis 922 (enquête de suivi) sélectionnés par randomisation dans l'enquête MMAS longitudinale. De nombreux traitements sont associés à la dysfonction érectile mais, après ajustement sur les co-morbidités, seuls les diurétiques non thiazidiques et les benzodiazépines restent significativement associés à la dysfonction érectile [13].

Par ailleurs les études randomisées contre d'autres médicaments ou contre placebo avec un nombre suffisant de patients sont rares, voire inexistantes, et certaines études n'ont pas toujours confirmé le rôle de certains traitements pourtant soupçonnés depuis de nombreuses années comme les bêtabloquants.

L'étude TOMHS, publiée en 1997, prospective, randomisée, en double aveugle, concerne 557 hommes de 45 à 69 ans hypertendus traités par placebo, diurétique thiazidique (chlortalidone), alphabloquant (doxazosine), inhibiteur de l'enzyme de conversion (enalapril), inhibiteur calcique (amlodipine) ou bêtabloquant (acebutolol). Cette étude conclut à la faible incidence de la dysfonction érectile chez les hypertendus traités : à 24 mois, seul le diurétique donne 
une incidence plus élevée que le placebo, mais à 48 mois aucune différence n'apparaît. Si une dysfonction érectile existait au début de l'étude elle s'améliore, surtout sous alphabloquant [22].

Franzen, également au cours d'une étude prospective randomisée en double aveugle contre placebo, ne retrouve pas d'influence du métoprolol (bêtabloquant) sur la sexualité [20].

A l'évidence certaines classes thérapeutiques ont tellement évoluées ces dernières années que leur mauvaise réputation "historique" mériterait d'être reconsidérée, d'autant que les mécanismes à l'origine de troubles de l'érection postmédicamenteux restent souvent inconnus.

\section{Insuffisance rénale chronique}

\section{a) Prévalence de la dysfonction érectile}

La prévalence de la dysfonction érectile est élevée chez les insuffisants rénaux en dialyse. Trois études récentes (Tableau 3) chiffrent cette prévalence entre 47,3 et 59,5\% (entre 43,6 et $60,8 \%$ en ce qui concerne la dysfonction érectile modérée ou sévère) $[10,40,44]$.

\section{b) Etiologies}

Les causes sont multiples : endocriniennes (hypogonadisme périphérique et hyperprolactinémie), neurologiques (neuropathies végétatives et périphériques), vasculaires (des facteurs de risque vasculaires sont souvent associés dans cette population : dans les séries respectives de Rosas [44] et de Neto [40], 97 et $96 \%$ des patients sont hypertendus, 39 et $26 \%$ diabétiques), médicamenteuses et psychologiques (anxiété, dépression, perte du travail, conflit conjugal) [9].

\section{c) Facteurs de risque}

La prévalence de la dysfonction érectile chez les insuffisants rénaux chroniques augmente avec l'âge, le diabète, un faible revenu, la non-utilisation des inhibiteurs de l'enzyme de conversion, des perturbations psycho-émotionnelles, un faible taux de cholestérol HDL [10, 40, 44].

\section{d) Influence de la transplantation rénale}

La transplantation rénale ne résout pas tous les problèmes. Malavaud chiffre la prévalence de la dysfonction érectile chez 271 transplantés à 55,7\% avec 3 facteurs de risque : l'âge, l'ancienneté de la dialyse et le caractère itératif des greffes [35].

\section{Facteurs socio-économiques et mode de vie}

Un mode de vie sédentaire favorise la dysfonction érectile alors que l'activité physique est au contraire protectrice. Derby le démontre dans l'enquête MMAS longitudinale (évaluation de l'incidence de la dysfonction érectile chez 593 hommes initialement sans dysfonction érectile ni cancer de prostate ni traitements pour une maladie cardiaque ou un diabète) [14].

De faibles niveaux d'études ou d'éducation, une baisse de revenus ou de faibles revenus mensuels sont également des facteurs de risque $[30,38]$ et, selon Aytac (enquête MMAS longitudinale), les cols "bleus" souffrent plus de dysfonction érectile que les cols "blancs" (OR ajusté $=1,55)$ [3].

Selon Laumann, le mariage protège de la dysfonction érectile $(\mathrm{OR}=1,72$ pour les célibataires, 1,6 pour les séparés, divorcés et veufs) [30]. Toutefois certains auteurs contestent l'influence des facteurs socio-économiques notamment du niveau d'éducation et d'études $[1,42]$ et du statut marital [42].

\section{Hormones}

Dans l'enquête MMAS initiale, seul un faible taux de SDHEA (sulfate de déhydroépiandrostérone) est corrélé avec la dysfonction érectile [17].

\section{Obésité}

Chung estime que l'obésité n'est pas un facteur de risque [12] en elle-même, mais pour Derby, dans l'enquête MMAS longitudinale citée au chapitre du mode de vie, l'incidence de la dysfonction érectile est plus élevée chez les patients initialement obèses (indice de masse corporelle $\geq 30$ ) quelque soit le devenir du poids au cours de la période d'observation de 8 ans [14].

\section{Symptômes du bas appareil urnaire}

Les symptômes du bas appareil urinaire sont souvent associés à la dysfonction érectile $[30,38]$. Braun relève $72 \%$ de patients souffrant de tels symptômes parmi les insuffisants érectiles contre $37,7 \%$ dans la population sans dysfonction érectile [7]. Frankel confirme cette association significative au cours d'une enquête menée dans une popu-

Tableau 3 : Prévalence de la dysfonction érectile chez les insuffisants rénaux chroniques.

\begin{tabular}{|c|c|c|c|c|c|c|}
\hline Auteur (année) & $\begin{array}{l}\text { Nombre } \\
\text { de patients }\end{array}$ & Age moyen & $\begin{array}{l}\text { Dysfonction } \\
\text { érectile (DE) }\end{array}$ & $\begin{array}{l}\text { DE sévère } \\
\text { et modérée }\end{array}$ & $\begin{array}{l}\text { DE sévère et } \\
\text { modérée } \\
\text { avant } 50 \text { ans }\end{array}$ & $\begin{array}{l}\text { DE sévère et } \\
\text { modérée après } \\
50 \text { ans }\end{array}$ \\
\hline Rosas (2001) [44] & 302 & 59,5 & $82 \%$ & $53 \%$ & $28 \%$ & $64 \%$ \\
\hline $\operatorname{Neto}(2002)[40]$ & 118 & 48 & $86,4 \%$ & $60,8 \%$ & $52,6 \%$ & $70,5 \%$ \\
\hline Cerqueira (2002) [10] & 119 & 47,3 & $57,9 \%$ & $43,6 \%$ & - & - \\
\hline
\end{tabular}


lation générale de 423 hommes de plus de 40 ans (dans une région du Royaume-uni) et dans une population ciblée de 1271 hommes de plus de 45 ans ayant consultés en urologie avec des symptômes du bas apparcil urinaire (dans 12 pays du monde) [19].

\section{Ethylisme}

L'éthylisme est un facteur de risque attesté par certains $[17,38]$ mais contesté par d'autres $[7,42]$.

\section{Etat de santé général}

Selon Laumann, un mauvais état général est un facteur de risque de dysfonction érectile $(\mathrm{OR}=2,02)$ [30].

\section{Cyclisme}

Grunbaum a récemment publié une revue de littérature sur l'influence du cyclisme sur la fonction érectile [23]. En effet cette pratique sportive serait responsable d'hypoesthésie périnéale et génitale et de dysfonction érectile. Des phénomènes compressifs nerveux et vasculaires sont certainement en cause, mais la physiopathologie n'est pas complètement connue. La durée et la fréquence de la pratique de même que le type de selle influent certainement sur les conséquences du cyclisme sur la fonction érectile. L'enquête MMAS initiale montre notamment qu'une pratique de moins de 3 heures par semaine serait protectrice $(\mathrm{OR}=0,61)$, confirmant l'intérêt d'une activité physique, alors qu'une pratique de plus de 3 heures par semaine favoriserait la dysfonction érectile $(O R=1,72)$ [37].

\section{Autres facteurs}

De nombreuses autres pathologies favorisent la dysfonction érectile ou en sont la seule étiologie : chirurgie pelvienne (cystoprostatectomie totale, prostatectomie radicale), irradiation pelvienne, neuropathies centrales (traumatismes médullaires, maladie de Parkinson, sclérose en plaques), fibrose des corps caverneux (après priapisme ou maladie de La Peyronie), pathologies rhumatismales, allergies, ulcères [33].

\section{CONCLUSIONS}

La reconnaissance des facteurs de risque de dysfonction érectile grâce à de nombreuses enquêtes épidémiologiques modifie notre approche de cette pathologie et nous conduit à proposer une information différente et des mesures préventives. Néanmoins la poursuite de travaux dans ce domaine est indispensable car il persiste de nombreuses inconnues :

- certains facteurs de risque ne sont pas identifiés avec certitude ;

- les mécanismes physiopathologiques de certains facteurs de risque ne sont pas élucidés ;
- l'intérêt de certaines mesures préventives n'est pas démontré.

Compte tenu de l'intérêt exceptionnel suscité par l'épidémiologie de la dysfonction érectile depuis seulement quelques années, certaines questions ne resteront probablement pas longtemps sans réponses.

\section{REFERENCES}

1. ANSONG K.S., LEWIS C., JENKINS P., BİLL J. : Epidemiology of erectile dysfunction : a community-based study in rural New York State. Ann. Epidemiol., 2000, $10: 293-296$.

2. ARAUJO A.B., JOHANNES C.B., FELDMAN H.A., DERBY C.A., McKiINLAY J.B. : Relation between psychosocial risk factors and incident erectile dysfunction: prospective results from the Massachusetts Male Aging Study. Am. J. Epidemiol., 2000, $152: 533-541$.

3. AYTAC I.A., ARAUJO A.B., JOHANNES C.B., KLEINMAN K.P., McKINLAY J.B. : Socioeconomic factors and incidence of erectile dysfunction: findings of the longitudinal Massachussetts Male Aging Study. Soc. Sci. Med., 2000, 51 : 771-778.

4. BARKSDALE J.D., GARDNER S.F. : The impact of first-line antihypertensive drugs on erectile dysfunction. Pharmacotherapy, 1999, $19: 573-581$.

5. BEHR-ROUSSEL D., CHAMIOT-CLERC P., BERNABE J. et al. : L'hypertension artérielle : facteur de risque de l'insuffisance érectile - $1^{\text {ère }}$ démonstration expérimentale. Communication au $96^{\mathrm{e}}$ Congrès de l'Association Française d'Urologie, 20 - 23 novembre 2002, Paris.

6. BORTOLOTTI A., FEDELE D., CHATENOUD L., et al. : Cigarette smoking : a risk factor for erectile dysfunction in diabetics. Eur. Urol., 2001, $40:$ 392-396.

7. BRALiN M., WASSMER G., KLOTZ T., REIFENRATH B., MATHERS M., ENGELMAN U. : Epidemiology of erectile dysfunction: results of the 'Cologne Male Survey'. Int. J. Impot. Res., 2000, 12 : 305-311.

8. BURCHARDT M., BURCHARDT T., BAtR L. et al. : Hypertension is associated with severe erectile dysfunction. J. Urol., 2000, $164: 1188-1191$.

9. CARSON C.C., PATEL M.P. : The epidemiology, anatomy, physiology, and treatment of erectile dysfunction in chronic renal failure patients. Adv. Ren. Replace. Ther., 1999, $6: 296-309$.

10. CERQUEIRA J., MORAES M., GLINA S. : Erectile dysfunction: prevalence and associated variables in patients with chronic renal failure. Int. J. Impot. Res., 2002, 14 : 65-71.

11. CHEW K.K., EARLE C.M., STUCKEY B.G., JAMROZIK K., KEO(iH E.J. : Erectile dysfunction in general medicine practice: prevalence and clinical correlates. Int. J. Impot. Res., 2000, 12 : 41-45.

12. CHUNG W.S., SOHN J.H., PARK Y.Y. : Is obesity an underlying factor in erectile dysfunction ? Eur. Urol., 1999, 36: 68-70.

13. DERBY C.A., BARBOUR M.M., HUME A.L., McKINLAY J.B. : Drug therapy and prevalence of erectile dysfunction in the Massachusetts Male Aging Study cohort. Pharmacotherapy, 2001, $21: 676-683$.

14. DERBY C.A., MOHR B.A., GOLDSTEIN I., FELDMAN H.A. 
JOHANNES CB, MCKinlay JB : Modifiable risk factors and erectile dysfunction : can lifestyle changes modify risk. Urology, $2000,56: 302-306$.

15. FEDELE D., COSCELLI C., CUCINOTTA D. et al. : Incidence of erectile dysfunction in Italian men with diabetes. J. Urol., $2001,166: 1368-1371$.

16. FEDELE D., COSCELLI C., SANTHUSANIO F. et al. : Erectile dysfunction in diabetic subjects in Italy. Gruppo Italiano Studio Deficit Erettile nei Diabetici. Diabetes Care, 1998, 21 : 19731977.

17. FELDMAN H.A., GOLDSTEIN I., HATZICHRISTOU D.G., KRANE R.J., McKINLAY J.B. : Impotence and its medical and psychosocial correlates: results of the Massachusetts Male Aging Study. J. Urol., 1994, 151 : 54-61.

18. FELDMAN H.A., JOHANNES C.B., DERBY C.A. et al. : Erectile dystunction and coronary risk factors: prospective results from the Massachusetts male aging study. Prev. Med., 2000, 30 : 328-338.

19. FRANKEL S.J., DONOVAN J.L., l'ETERS T.I. et al. : Sexual dysfunction in men with lower urinary tract symptoms. J. Clin. Epidemiol., 1998, $51: 677-685$.

20. FRANZEN D., METHA A., SELFI:RT N., BRAUN M., HOPP H.W. : Effects of beta-blockers on sexual performance in men with coronary heart disease. A prospective, randomized and double blinded study. Int. J. Impot. Res., 2001, 13 : 348-351.

21. GREENSTEIN A., CHEN J., MILLER H., MAT /KIN H., VILLA Y., BRAF Z. : Does severity of ischemic coronary disease correlate with erectile function? Int. J. Impot. Res., 1997, 9:123-126

22. GRIMM R.H. Jr, GRANDITS G.A., PRINEAS R., et al. : Longterm effects on sexual function of five antihypertensive drugs and nutritional hygienic treatment in hypertensive men and women. Treatment of Mild Hypertension Study (TOMHS). Hypertension, 1997, $29: 8-14$.

23. GRUNBAUM A., KHALEEG-UR-REHMAN, CARRIER S. : Bicycling and erectile dysfunction : a review of the literature. $J$. Sex. Reprod. Med., 2002, 2 : 75-79.

24. GUAY A.T., PEREZ J.B., HEAT (i.J. : Cessation of smoking rapidly decreases erectile dysfunction. Endoc. Pract., 1998, 4. 23-26.

25. HAKIM L.S., GOLDSTEIN I. : Diabetic sexual dysfunction. Endocrinol. Metab. Clin. North Am., 1996, 25 : 379-400.

26. JENSEN J., LENDORF A., STIMPLL H., FROST J., IBSEN H., ROSENKILDE $\mathrm{P}$. : The prevalence and etiology of impotence in 101 male hypertensive outpatients. Am. J. Hypertens., 1999, 12 : 271-275.

27. JOHANNES C., ARAUJO A.B., FELDMAN H.A., DERBY C.A., McKINLAY J.B. : Incidence of erectile dystunction in men 40 to 69 years old : longitudinal results from the Massachusetts Male Aging Study. J. Urol., 2000, 163: 460-463.

28. KIM S.W., PAICK J., PARK D.W., CHAE I., OH B. : Potential predictors of asymptomatic ischenic heart disease in patients with vasculogenic erectile dysfunction. Urology, 2001, 58 : 441445.

29. KLEIN R., KLIIN B.E., LEE K.E., MOSS S.E., CRUICKSHANKS K.J.. : Prevalence of self-reported erectile dysfunction in people with long-term IDDM. Diabetes Care, 1996, 19: 135141.

30. LAUMANN E.O., PAIK A., ROSINN R.C. : Sexual dysfunction in the United States : prevalence and predictors. J.A.M.A., 1999, 281 : 537-544.

31. LEE I.C., SURRIDGE D., MORALES A., HEATON J.P. : The prevalence and influence of significant psychiatric abnormalities in men undergoing comprehensive management of organic ercetile dysfunction. Int. J. Impot. Res., 2000, $12: 47-51$.

32. LERICHE A., JAUDINOT E.O., SOI.ISSSE DE GENDRE A. : Enquête sur la prévalence des troubles de l'érection et leur prise en charge chez des patients à risque suivis en consultation de médecine générale. Communication au $94^{\text {ème }}$ Congrès de l'Association française d'urolugie, Paris, 15-19 Novembre 2000, Résumé 03.

33. LEWIS R.W. : Epidemiology of erectile dysfunction. Urol. Clin. North Am., 2001, $28:$ 209-216.

34. McVAY K.T., CARRIER S., WESSliLLS H. : Smoking and erectile dysfunction : evidence based analysis. J. Urol., 2001, $166: 1624-1632$.

35. MALAVAUD B., ROSTAING L., RISCHMANN P., SARRAMON J.P., DURAND D. : High prevalence of erectile dysfunction after renal transplantation. Transplantation, 2000, $69: 2121$ 2124.

36. MANNINO D.M., KLEVENS R.M., ILANDERS W.D. : Cigarette smoking : an independent risk factor for impotence ? Am. J. Epidemiol., 1994, 140 : 1003-1008.

37. MARCEAU L., KI.EINMAN K., GOI.DSTEIN I., McKINLAY J. : Does bicycling contribute to the risk of erectile dysfunction ? Results from the Massachusetts Male $\Lambda$ ging Study (MMAS). Int. J. Impot. Res., 2001, 13 : 298-302.

38. MARTIN-MORALES A., SANCHEZ-CRUZ J.J., SAENZ DE TEJADA I., RODRIGUEZ-VELA I.., JIMENEZ-CRUZ J.F., BURGOS-RODRIGUEZ R. : Prevalence and independent risk factors for erectile dysfunction in Spain : results of the Epidemiologia de la Disfunction Erectil Masculina Study. J. Lrol., $2001,166: 569-574$.

39. MEINHARDT W., KROPMAN R.F., VERMEIJ P., NIJIHOLT A.A., ZWARTENDIJK J. : The influence of medication on erectile function. Int. J. Impot. Res., 1997, $9: 17-26$.

40. NETO A.F., DE FREITAS RODRIGUI:S M.A., SARAIVA FITTIPALDI J.A., MOREIRA E.D. Jr : The epidemiology of erectile dysfunction and its correlates in men with chronic renal failure on hemodialysis in Londrina, southern Brazil. Int. J. Impot. Res., 2002.14 Suppl 2 : 19-26.

41. O'KANE P.D., JACKSON G. : Erectile dysfunction : is there silent obstructive coronary artery discase ? Int. J. Clin. Pract., 2001, $55: 219-220$.

42. PARAZZINI F., MENCHINI FABRIS F., BORTOLLI A. : Frequency and determinants of erectile dysfunction in Italy. Eur. Urol., 2000, $37: 43-49$.

43. PINNOCK C.B., STAPIETON A.M., MARSHALI V.R. : Erectile dysfunction in the community: a prevalence study. Med. J. Aust., 1999, $171:$ 353-357.

44. ROSAS S.E., JOFHE M., FRANKLIN E. et al. : Prevalence and determinants of erectile dysfunction in hemodialysis patients. Kidney Int., 2001, 59 : 2259-2266.

45. SAIRAM K., KLI.INSKAYA E., BOISTEAD G.B., HANBLRY D.C., McNICHOLAS T.A. : Prevalence of undiagnosed diabetes mellitus in male erectile dysfunction. Brit. J. Urol. Int., $2001,88: 68-71$. 
46. SCHACHTER M. : Erectile dysfunction and lipid disorders. Curr. Med. Res. Opin., 2000, 16 Suppl 1 : 9-12.

47. SIU S.C., LO S.K., WONG K.W., IP K.M., WONG Y.S. : Prevalence of and risk factors for erectile dysfunction in Hong Kong diabetic patients. Diabet. Med., 2001, 18: 732-738.

48. SULLIVAN M.E., MILLER M.A., BELL C.R. et al. : Fibrinogen, lipoprotein (a) and lipids in patients with erectile dysfunction. A preliminary study. Int. Angiol., 2001, 20: 195-199.

49. SULLIVAN M.E., THOMPSON C.S., DASHWOOD M.R. et al.: Nitric oxide and penile erection: is erectile dysfunction another manifestation of vascular disease ? Cardiovasc. Res.. 1999, $43: 658-665$.

50. TENGS T.O., OSGOOD N.D. : The link between smoking and impotence : two decades of evidence. Prev. Med., 2001, 32 : 447-526.

51. WEI M., MACERA C.A., DAVIS D.R., HORNUNG C.A., NANKIN H.R., BLAIR S.N. : Total cholesterol and high density lipoprotein cholesterol as important predictors of erectile dysfunction. Am. J. Epidemiol., 1994, 140 : 930-937.

\author{
ABSTRACT \\ Epidemiology of erectile dysfunction (part 2). \\ Risk factors
}

Dominique DELAVIERRE

Epidemiological studies in the general population or target populations in several countries in the world have revealed a large number of risk factors for erectile dysfunction: diabetes mellitus, hypertension, smoking, dyslipidaemia, cardiovascular diseases, psychological disorders, certain medications, chronic renal failure, socioeconomic factors and lifestyle, obesity, lower urinary tract symptoms, poor health and bicycling.

Cardiovascular risk factors are predictors of erectile dysfunction and erectile dysfunction is now considered to be a manifestation of vascular disease.

Further studies are necessary to establish the pathophysiological mechanisms of certain risk factors and the possible value of preventive measures.

Key-Words: epidemiology, prevalence, erectile dysfunction, impotence 